\title{
Development of Cylindrical Drift Chamber for COMET Phase-I
}

\author{
T.S. Wong ${ }^{* 1}$, Y. Kuno ${ }^{1}$, A. Sato ${ }^{1}$, H. Sakamoto ${ }^{1}$, H. Yoshida ${ }^{1}$, C. $\mathbf{W u}^{2,3}$, H. Katayama ${ }^{1}$ \\ Y. Nakazawa ${ }^{1}$ and the COMET collaborators \\ ${ }^{1}$ Department of Physics, Osaka University, \\ ${ }^{2}$ Institute of High Energy Physics (IHEP) \\ ${ }^{3}$ NanJing University, China, \\ E-mail: samwong@kuno-g.phys.sci.osaka-u.ac.jp
}

In the experiment, CDC Prototype II and III were stringed with $\phi 25 \mu \mathrm{m}$ and $\phi 30 \mu \mathrm{m}$ tungsten anode wire coated with gold, respectively. Three He-based gas candidates with $\mathrm{iC}_{4} \mathrm{H}_{10}, \mathrm{CH}_{4}$ and $\mathrm{C}_{2} \mathrm{H}_{6}$ were studied. For the setup, two scintillators were used as the trigger and four tracking chambers were used to reconstruct electron beam tracks in 3 dimension. The data was acquired by using four Belle II RECBE boards. With different setting of applied high voltage and signal threshold, the detector response with efficiency more than $95 \%$ and spatial resolution less than $\sigma=200 \mu \mathrm{m}$ for each prototype chamber were confirmed. The result of this experiment has lead us to finalize the parameters of the CDC in Phase-I. In this paper, the analysis method and result will be described in details.

Flavor Physics \& CP Violation 2015

May 25-29, 2015

Nagoya, Japan

${ }^{*}$ Speaker. 


\section{Introduction}

COMET is a J-PARC E21 experiment aiming at the search of neutrinoless $\mu^{-}-e^{-}$conversion with a single-event of sensitivity of $3 \times 10^{-15}$ for Phase-I and $3 \times 10^{-17}$ for Phase-II which is a factor of 10,000 better than the current upper limit[1]. In COMET Phase-I, the Cylindrical Drift Chamber (CDC) is the main part of the detection system. To achieve $10^{-15}$ single-event of sensitivity, COMET CDC is required to have momentum resolution about $200 \mathrm{keV} / \mathrm{c}$. Hence, Helium-based gas mixture will be used to suppress the multiple scattering so that high momentum resolution measurement can be accomplished. Since momentum resolution is directly proportional to the spatial resolution, a test of detector performance should be carried out. To test prototypes of the CDC (fig.1), an experiment was performed at Research Center for Electron Photon Science of Tohoku University and Electron Beam with momentum at 460MeV/c in December, 2014. The purpose of this study was to evaluate the detector performance of the prototype CDC.

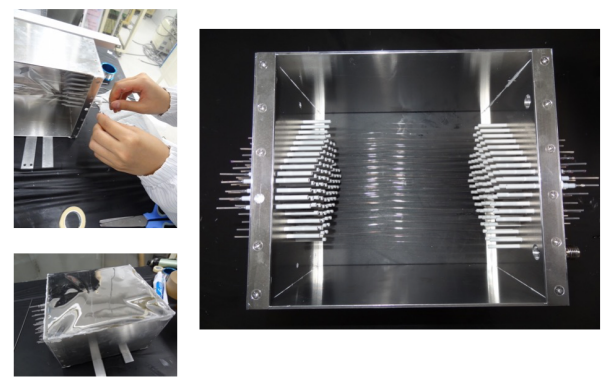

(a)

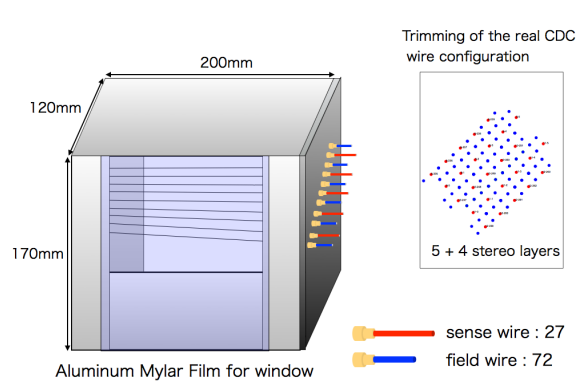

(b)

Figure 1: Schematic and Construction Prototype II and III

\section{Beam Test}

\subsection{Goals of Beam test}

In the beam test, CDC prototype II and III were constructed with anode wire $\phi 25 \mu \mathrm{m}$ and $\phi 30 \mu \mathrm{m}$, respectively, with stereo angle +-65 mrad. The anode wires were made of tungsten and coated with gold. The characteristic of prototype II and III are summerized in table 1. For the gas types, three He-based gas candidates with $\mathrm{iC}_{4} \mathrm{H}_{10}, \mathrm{CH}_{4}$ and $\mathrm{C}_{2} \mathrm{H}_{6}$ were selected. In order to estimate the performance of the detector for each gas mixture, the following items were studied. (1) Gas gain (gain curve),(2) Position-time relation (XT-curve),(3) Spatial resolution,(4) Hit efficiency (plateau curve).

\subsection{Setup and Trigger Logic}

Momentum of electron beams was set to $460 \mathrm{MeV} / c$ (Magnet current was set to 230A). The setup of the beam test is shown in fig.2a. At the upstream and downstream, scintillator counters 
were installed. Each scintillator was read out by 1-inch PMT. Followed by the scintillators, tracking chambers of $\mathrm{X}$ and $\mathrm{Y}$ directions were installed. CDC Prototype II and III were located at the center of the setup. Each prototype and tracking chamber were then read out by 48ch RECEBE board. Next to the setup, the gas system, NIM modules and DAQ systems are stationed. And the signal was sent to the computer at the control room and the signal quality was online-monitored. Trigger signals were made by using the conincidence of PMTs' signals. To avoid overloading of the RECBE boards and DAQ, a VETO of $100 \mu$ s was uilitzied. The trigger signals were then delayed by $700 \mathrm{~ns}$ and sent to 4 readout boards. The figure is shown in fig. $2 \mathrm{~b}$.

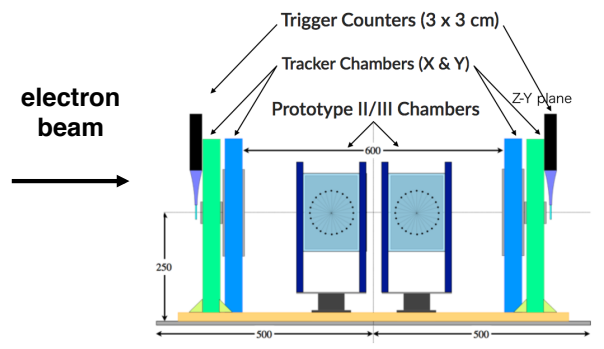

(a)

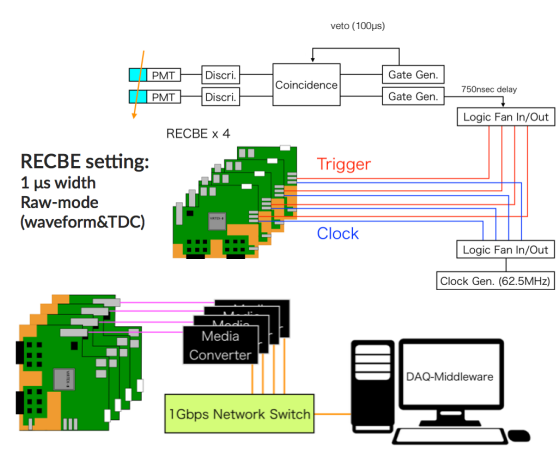

(b)

Figure 2: (a) Setup at the beam test. (b) Trigger Logic at the beam test.

\begin{tabular}{l|l|l}
\hline \hline Prototype Number & II & III \\
\hline \hline Anode Wire & $\phi 25 \mu \mathrm{m} \mathrm{W}$ with Au & $\phi 30 \mu \mathrm{m} \mathrm{W}$ with Au \\
Cathode Wire & $\phi 80 \mu \mathrm{m} \mathrm{Al}$ & $\phi 80 \mu \mathrm{m} \mathrm{Al}$ \\
Cell size & $16 \times 16 \mathrm{~mm}$ & $16 \times 16 \mathrm{~mm}$ \\
\hline \hline
\end{tabular}

Table 1: The specification of prototype II and III

\section{Analysis and Result}

\subsection{Procedure of analysis}

To get the drift distance, X-T relation of tracking chamber's geometry simulated by garfield simulation was used and input for getting drift distance again. Three times of iteration were carried out to make sure the X-T relation converaged. With the condition of 8 hits in the tracking chambers and limitation of boundary hit in one cell, the event efficiency is dropped to around 50\%. After the cutevents, 4 hit cells in the $\mathrm{X}$ or $\mathrm{Y}$ tracking chambers were used for $\chi^{2}$ fitting. A 3 dimension straight track can be calculated and then used to studied the performance of prototypes. The tracks with $\chi^{2}$ in tracking chamber $\mathrm{X}$ smaller were discarded. The event efficiency is about $25 \%$ finally. The $\chi^{2}$ distribution and selected beam spot (X-Y plane) at upstream tracker are shown in fig.3a and 
fig. $3 \mathrm{~b}$ respectivily. The closest approach to each signaled anode wire was then obtained to get each wire's X-T relation (fig.3c). By fitting the X-T relation bin by bin filled in the histogram, the spatial resolution (fig.3d) can then be obtained by the difference of hit distance and fit distance. The hit efficiency is then calculated by counting the hits within $600 \mu \mathrm{m}$. Finally, this process was repeated for different high voltages and thresholds.

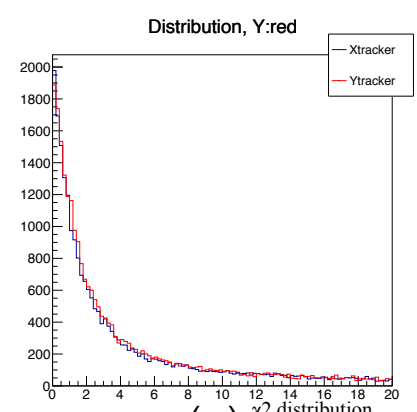

(a)

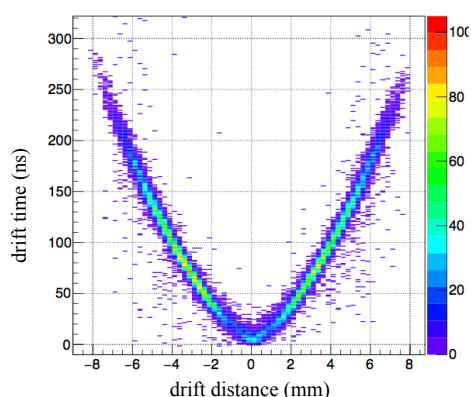

(c)

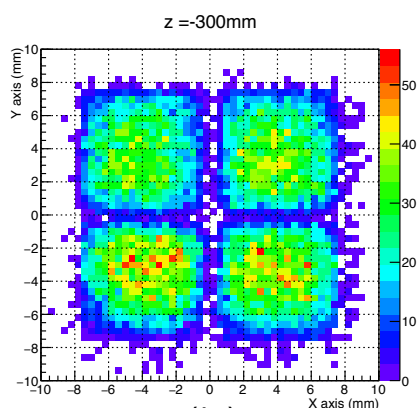

(b)

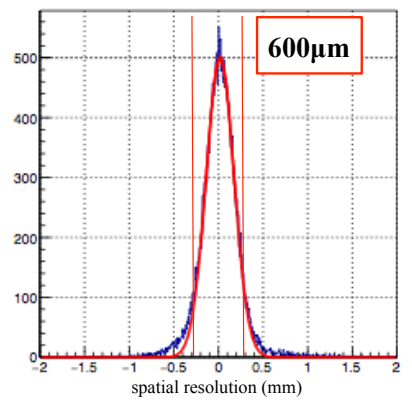

(d)

(a)

Figure 3: (a) $\chi^{2}$ distribution of $\mathrm{X}$ and $\mathrm{Y}$ tracking chambers, (b) Beam spot of selected events at upstream tracker, (c) Typical drift time and distance relation (X-T relation) of centre wire, (d) Typical spatial resolution $(\sigma)$ of centre wire

\subsection{Gas Gain}

Knowing the number of primary ions generated by an electron beam in a cell and the energy deposit by G4beamline simulation. The calculation of gas gain and the measured gas gain curve are shown in reference[2].

\subsection{Spatial resolution and Hit Efficiency curve}

The asymmetric feature of the spatial resolution in fig. $3 \mathrm{~d}$ reviews the fact that the spatial resolution depends on the drift distance. The spatial resolution, including tracking errors, are done in each drift distance from $0 \mathrm{~mm}$ to $8 \mathrm{~mm}$ in $1 \mathrm{~mm}$ step. Averageing the spatial resolution in 8 regions, the spatial resolution was obtained. Hit efficiency is defined as the ratio of the number of events within $0.6 \mathrm{~mm}$ of residuals to the number of reconstructed events, which is dependent on drift distance as well. Similarily, hit efficiency was obtained. Spatial resolution and Hit efficiency 
as a function of voltage for Helium-Ethane gas are shown in fig.3. Results for other gas mixture can be checked in reference[3].

The best effciencies for each gas mixture are listed out in table 2. Efficiency of $\mathrm{He}_{\mathrm{iC}} \mathrm{iC}_{4} \mathrm{H}_{10}$ and $\mathrm{He}: \mathrm{C}_{2} \mathrm{H}_{6}$ are about $95 \%$ whereas $\mathrm{He}: \mathrm{CH}_{4}$ is smaller than $95 \%$. It is found that efficiency drop found at higher $\mathrm{HV}$ due to crosstalk and improper shielding of sense cable at readout board side.

\begin{tabular}{l|l|l|l|l}
\hline \hline Gas Type & Wire Size $(\mu \mathrm{m})$ & High Voltage(V) & Efficiency (\%) & Spatial Resolution $(\mu \mathrm{m})$ \\
$\mathrm{He}: \mathrm{iC}_{4} \mathrm{H}_{10}(90: 10)$ & 25 & $1850-1900$ & $94-96 \%$ & $170-170$ \\
& 30 & $1850-1950$ & $95-95 \%$ & $170-166$ \\
$\mathrm{He}: \mathrm{C}_{2} \mathrm{H}_{6}(50: 50)$ & 25 & $2300-2600$ & $95-96 \%$ & $176-173$ \\
& 30 & $2350-2600$ & $95-96 \%$ & $183-180$ \\
$\mathrm{He}: \mathrm{CH}_{4}(73: 27)$ & 25 & $2250-2300$ & $93-92 \%$ & $169-174$ \\
& 30 & $2300-2350$ & $92-92 \%$ & $167-169$ \\
\hline \hline
\end{tabular}

Table 2: Best Efficiency and Spatial resolution of different types of gas mixture for Prototype II and III

\section{Conclusion and Discussion}

Spatial resolution are measured to be less than $200 \mu \mathrm{m}$ including tracking errors for all gas mixtures and both prototype chambers. Efficiencies of about $95 \%$ were measured for all gas mixtures and both prototype chambers. These results suggests that both $\phi 25 \mu \mathrm{m}$ and $\phi 30 \mu \mathrm{m}$ can be used as anode wire. Another conisderation is the size of field wire. Since increasing the size of field wire allows us to increase the applied high voltage so as to reduce the effect of aging effect.[4]

Two kinds of crosstalks have been observed in the beam test. One of them was occurred across the channels of the same ASD in RECBE readout board. The crosstalk-to-signal is bipolar and is around $-1.0 \%$. The other type of crosstalk is intrinsic and it is caused by the positive ions induction and it was found to be around -3.0\%[4]. There was a phenomenon observed in the beam test and we named it the oscillation after the signal. One view is that the grounding and shielding of the prototype was not well prepared.

\section{References}

[1] COMET collaboration, Technical Design Report, http://comet.kek.jp/Documents_files/PAC-TDR-2014/PAC-Review-20141110.pdf

[2] Y. Nakazawa et al., COMET Phase-I CDC Prototype Beamtest 1, 70th JPS meeting at Waseda University, Mar. 212015

[3] T.S Wong et al., COMET Phase-I CDC Prototype Beamtest 2, 70th JPS meeting at Waseda University, Mar. 212015

[4] S. Uno et al., Study of a Drift Chamber Filled with a Helium-Ethane Mixture, Nucl. Instr. Meth A330(1993)55-63 